\title{
Schwannoma trigeminal intracraneal con extensión a la fosa infratemporal, espacio parafaríngeo, órbita, seno maxilar y fosa nasal. A propósito de un caso
}

\author{
J.A. Santos-Franco; A. Barragán*; R. Mercado-Pimentel; I. Ortiz-Velásquez; C. García-Pastor; E.I. Barquet-Platón**; \\ C. Pane-Pianesse* y S. Gómez-Llata \\ Servicio de Neurocirugía. Servicio de Neuro-otología* Unidad de Cuidados intensivos**. Instituto Nacional de Neurología y Neurocirugía \\ "Manuel Velasco Suárez". México.
}

Resumen:

Los schwannomas constituyen del 8 al $\mathbf{1 0 \%}$ de los tumores intracraneales. Su asiento principal es la rama vestibular del VIII nervio craneal, siendo los trigeminales de escasa frecuencia. Presentamos el caso de una paciente admitida en el Instituto Nacional de Neurología y Neurocirugía "Manuel Velasco Suárez" de la ciudad de México con una masa en la fosa infratemporal con extensión importante a estructuras vecinas. Los schwannomas con extensión a la fosa infratemporal son raros. Revisamos la anatomía de la fosa infratemporal, los accesos quirúrgicos hacia dicha región, analizamos la serie de casos junto al nuestro y proponemos una modalidad quirúrgica distinta.

PALABRAS CLAVE: Schwannoma del trigémino. Fosa infratemporal

Intracraneal trigeminal schwannoma with extension to infratemporal fossa, parapharingeal space, orbit, maxillary sinus and nasal fossa

\section{Summary}

Schwannomas reach 8 to $10 \%$ of all intracranial tumors. Most originate at the vestibular root of VIII cranial nerve, but trigeminal tumors are infrequent. We present the case of a patient admitted at the National Institute of Neurology and Neurosurgery "Manuel Velasco Suárez" (Mexico City) with a mass occupying the infratemporal fossa with involvement of nearby structures. Schwannomas with extension to the infratemporal fossa are rare. We review the anatomy of this region, the surgical approaches, which have been used and propose a different approach.

KEY WORDS: Trigeminal Schwannoma. Infratemporal fossa.

Recibido: 26-02-03. Aceptado: 20-01-04
Introducción

Los schwannomas trigeminales son poco frecuentes, en relación a los vestibulares, y su localización puede variar ampliamente, debido a la distribución del nervio en sus tres ramas, involucrando diferentes regiones tanto intracraneales como extracraneales. La extensión hacia la fosa infratemporal ha sido poco descrita y puede constituir un reto diagnóstico y terapéutico.

\section{Caso clínico}

Mujer de 39 años con historia de trece años de cefalea progresiva en intensidad y, desde dos años antes de su ingreso a nuestra institución, con obstrucción nasal y anosmia derechas. Durante todo su padecimiento presentó proptosis progresiva del ojo derecho, junto con diplopia en la mirada extrema. La exploración física reveló proptosis derecha, con leve asimetría facial por aumento de volumen a nivel de mejilla ipsilateral. La rinoscopía mostró abombamiento septal con convexidad izquierda y una lesión tumoral en el área coanal derecha. La exploración neurológica fue normal.

La tomografía computada (TC) (figura 1) demostró una lesión hipodensa, ovoidea, ocupando la fosa infratemporal, seno esfenoidal, espacio parafaríngeo y fosa nasal derechos, provocando compresión y desplazamientos rostral de la pared caudal del seno maxilar, caudal de la apófisis pterigoides y dorsal del piso de la órbita, contactando incluso con el clivus; tras la administración de material de contraste mostró escasa captación. En la imagen de resonancia magnética (IRM) (figura 2) se mostró hipointensa en T1 e hiperintensa en T2, realzándose intensamente con gadolinio. Sus bordes aparecían bien definidos y, además de los desplazamientos ya escritos de la TC, se evidenció moldeamiento del paladar blando y duro y del septum nasal. También observamos adelgazamiento y deformidad

Abreviaturas: AMI: arteria máxilar interna. EPF: espacio parafaringeo. FIT: fosa infratemporal. FPP: fosa pterigopalatina. RM: Resonancia magnética. TAC: Tomografía computarizada. 


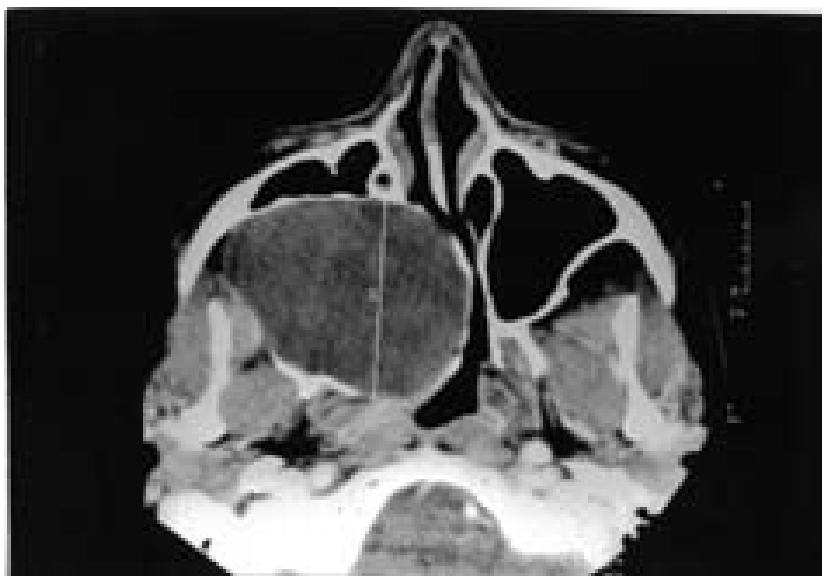

Figura 1 a. TC axial que muestra la lesión ocupando el espacio parafaríngeo y la rinolfaringe, desplazando rostralmente al seno maxilar.

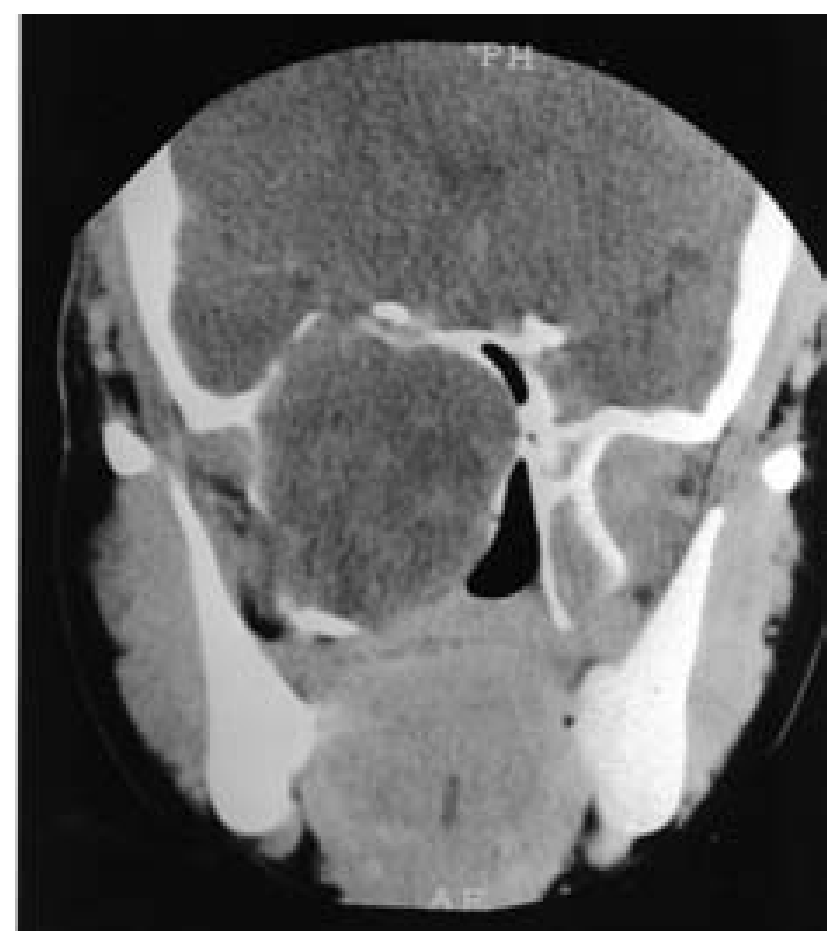

Figura 1 b: TC coronal en la que se ve claramente la lesión con erosión del piso medlio derecho y de la apófisis pterigoides ipsilateral.

del piso medio, lo cual hacía difícil determinar si la lesión se originaba en dicha área, o era una extensión desde la fosa infratemporal. Por lo tanto, los diagnósticos probables fueron de neurinoma y papiloma invertido del seno esfenoidal.

La paciente fue sometida a cirugía mediante incisión sublabial con abordaje intercartilaginoso centrofacial transmaxilar, replegamiento del colgajo miocutáneo y abordaje transmaxilar. Se halló una tumoración de bastante consistencia encapsulada (como tela de hule) de coloración grisácea, escasamente vascularizada que desplazaba estructuras vecinas. Se realizó la exéresis intracapsular de la lesión, sin incidentes. El estudio histopatológico reveló schwannoma (figura 3). El postoperatorio cursó sin complicaciones y a los tres meses la paciente se encuentra asintomática con mejoría notable de la proptosis. La TC de control mostró exéresis completa de la lesión (figura 4).

\section{Discusión}

Los shwannomas son tumores de lento crecimiento. El $60 \%$ se originan en el neurilema de los nervios craneales, principalmente en la zona de entrada de la raíz, también denominada de Obersteiner-Redlich. En esta zona se realiza la transición de la mielina central con la periférica y se ubica entre 8 y $12 \mathrm{~mm}$ del origen aparente del VIII par craneal, y de $0,8 \mathrm{~mm}$ y $2,2 \mathrm{~mm}$ de los nervios VII y V respectivamente $^{29}$.

Los nervios sensitivos son especialmente afectados, mientras que la afección de los motores suele asociarse a neurofibromatosis ${ }^{1}$. En general, los shwannomas constituyen del $8 \%$ a $10 \%$ de los tumores intracraneales y su asiento principal es la división superior de la rama vestibular del VIII nervio craneal y en escasa frecuencia, en orden descendente, derivan del V, IX, X y VII nervios craneános ${ }^{3,26}$. Se han descrito orígenes intracraneanos extremadamente raros, sin involucrar a nervios craneales ${ }^{10,15,21}$

Los originados en el trigémino representan del 0.8 hasta el $8 \%$ de todos los shwannomas intracraneanales ${ }^{26}$. Hasta la actualidad, en la literatura en inglés, se han reportado alrededor de 428 casos desde la primera descripción en 1849 hecha por $\mathrm{Smith}^{3,26}$. Estos tumores pueden aflorar de la raíz nerviosa, del ganglio de Gasser o de cualquiera de las tres divisiones del nervio trigémino; por lo tanto, pueden ocupar los pisos posterior y/o medio de la base del cráneo e incluso compartimentos extracraneales, confundiéndose en muchas ocasiones con tumores primarios de dichas regiones $^{2-4,7,12,13,16-20,23-25,29,31}$. Por lo tanto, Yoshida y Kawase ${ }^{31}$, los clasifican en 6 tipos: 1) $M$, a los localizados en la fosa media, ya sea que se hayan originado del ganglio de Gasser o de algunas de sus ramas en la pared del seno cavernoso; 2) $P$, a los que se originan de la raíz del trigémino y por consiguiente se localizan en la fosa posterior; 3) $E$, a los originados extracranealmente de alguna de las divisiones del V par; 4) MP, a los tumores que se encuentran en la fosa media y la posterior; 5) $M E$, a los localizados en el piso medio con extensión extracraneal; y, 6) $M P E$, a los que se ubican en los tres compartimentos. El caso aquí descrito pertenece al tipo $M E$, donde, los mismos autores, con fines de estrategia quirúrgica, dividen al espacio extracraneal en dos subgrupos: 1) Los que ocupan la órbita; y 2) los que invaden la fosa infratemporal y la fosa pterigopalatina. 


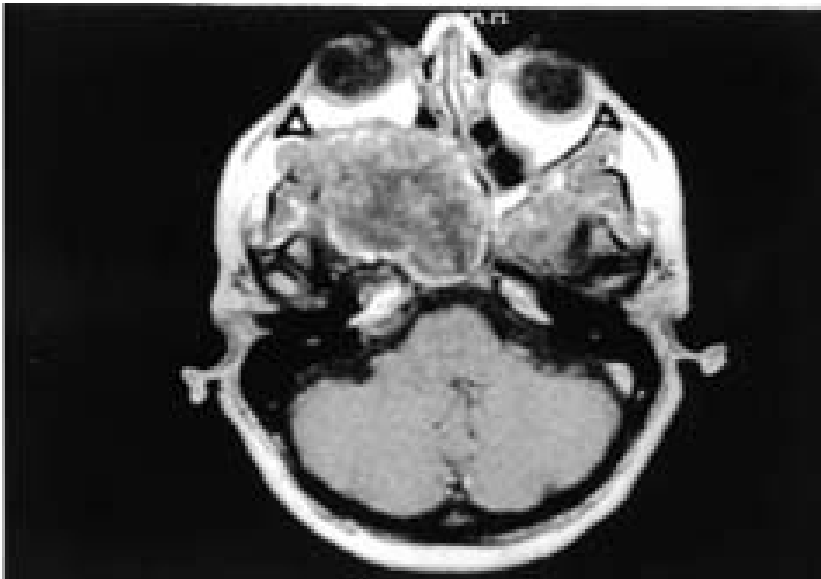

Figura 2 a. RM axial ponderada en T1 en la que se observa la afectación de la rinofaringe, la órbita y parte del piso medio de la base craneal.

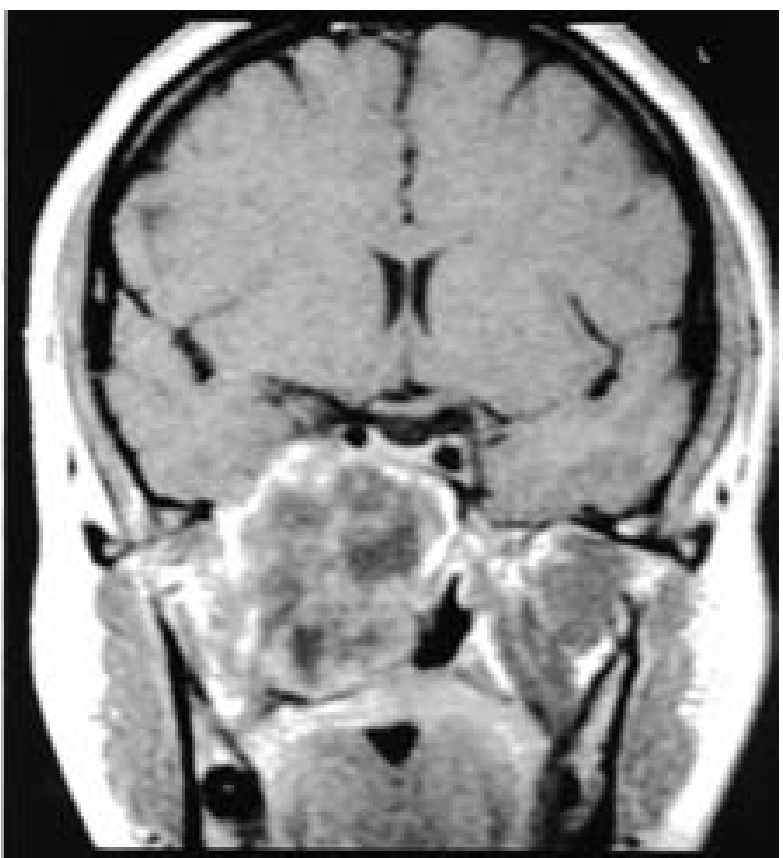

Figura 2 b. RM coronal en T1. Se observa que contacta con el lóbulo temporal derecho, desplaza dorsalmente a la carótida intracavernosar y se dirige ventralmente a la fósa infratemporal.

En la literatura mundial se han descrito 26 casos previos de shwannomas con extensión hacia fosa infratemporal (tabla 1). La fosa infratemporal se encuentra limitada rostralmente por la cara posterolateral del maxilar superior, rostromedialmente por la apófisis pterigoides, lateralmente por la rama ascendente del maxilar inferior y caudalmente por la porción timpánica del temporal y la apófisis estiloides. Dorsalmente se encuentra cubierta por la cara ventral del ala mayor del esfenoides, a nivel de los forámenes oval

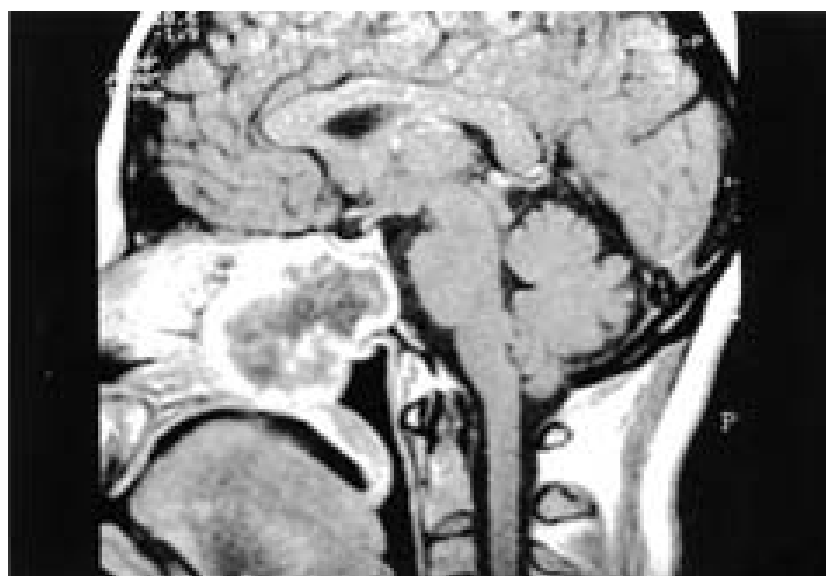

Figura 2 c. RM sagital en T1 donde se puede ver que la lesión llega a contactar con el paladar duro en su cara dorsal.

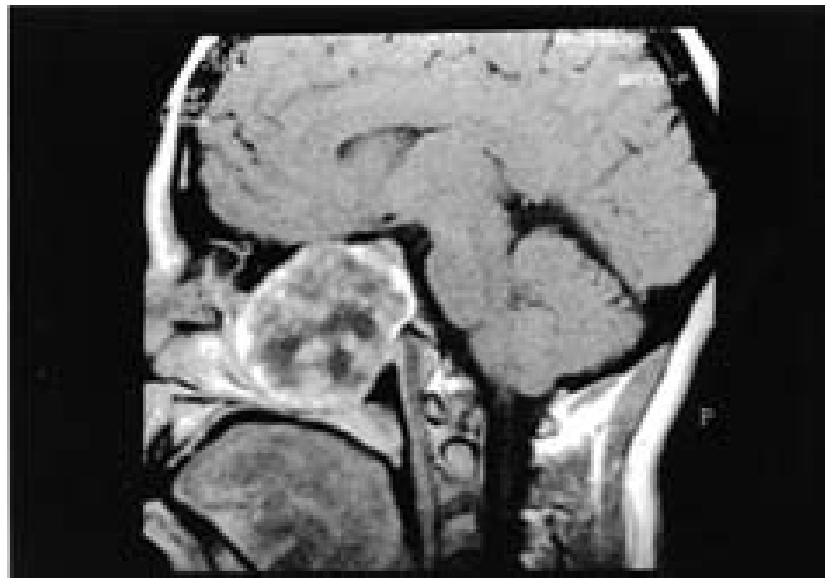

Figura 2 d. RM sagital en una vista paramediana derecha. Se evidencia el origen paraselar del tumor.

y redondo menor, y caudalmente por la porción escamosa del hueso temporal. Las caras dorsolateral, caudomedial de la fosa infratemporal no cuentan con paredes óseas. Los músculos pterigoideos medial y lateral cruzan, respectivamente, las partes ventral y dorsal de la fosa; el ligamento esfenomandibular desciende desde la espina esfenoidal hasta la língula del forámen mandibular. La arteria maxilar interna (AMI) entra en la fosa entre la rama ascendente de la mandíbula y el ligamento esfenomandibular, cerca del borde de la apófisis condilar mandibular.

En la fosa intratemporal (FIT), la arteria maxilofacial interna (AMI) da sus ramas auricular profunda, timpánica anterior, meníngea media y su accesoria, alveolar inferior, temporal profunda, pterigoidea, maseterina y bucal. El plexo venoso pterigoideo también se ubica en la FIT, y en su parte profunda se comunica con el seno cavernoso a través de venas emisarias; sin embargo su drenaje principal se realiza hacia la vena yugular interna a través de la vena 


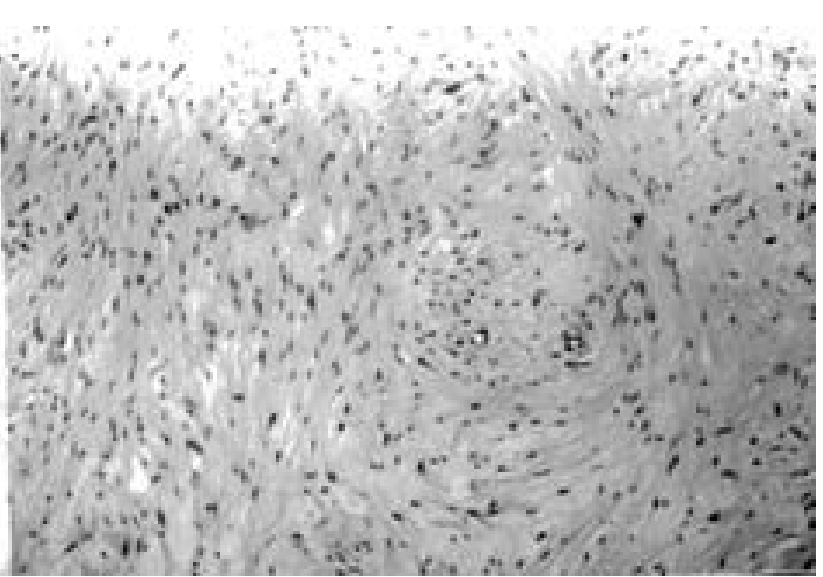

figura 3 a. Detalle histopatológico.

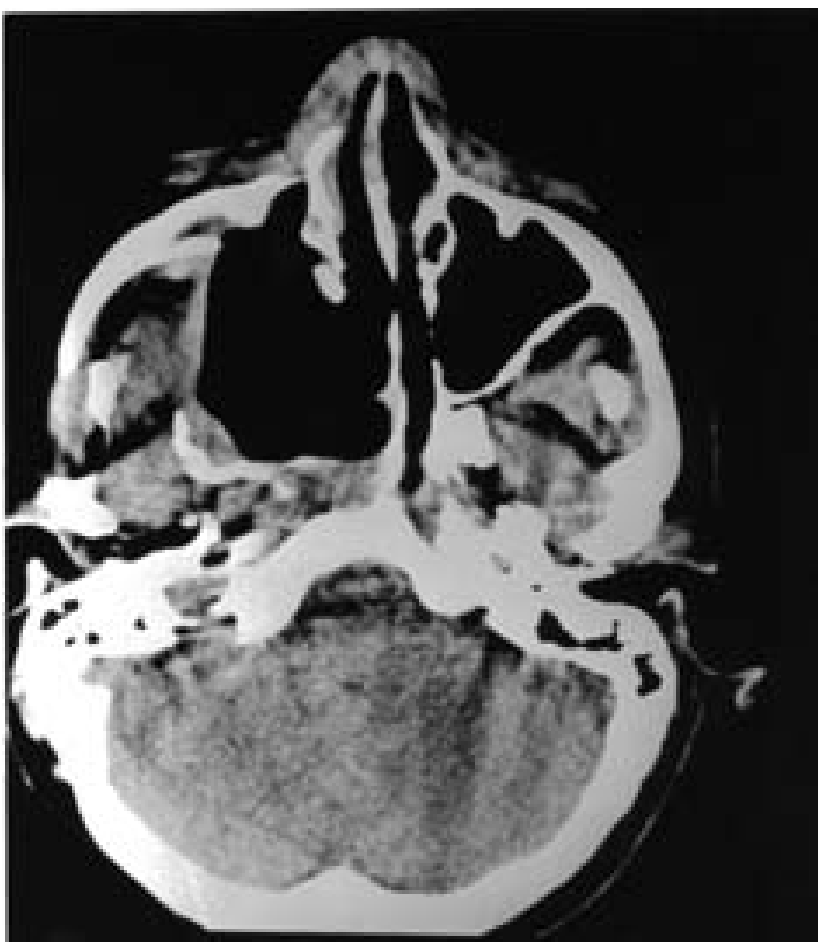

Figura 4 a. TC axial de control postoperatorio con ausencia aparente de la lesión.

maxilar. El nervio maxilar inferior (V3) ingresa en la fosa intratemporal (FIT) a través del agujero oval y se divide en dos troncos, uno anterior y otro posterior; del primero emergen los nervios temporal profundo, maseterino y bucal; del posterior los nervios lingual, alveolar inferior y auriculotemporal ${ }^{6,12,14,30,31}$. Algunos autores incluyen al espacio parafaríngeo dentro de la fosa infratemporal ${ }^{6,14}$. El espacio parafaríngeo (EPF) se describe como una pirámide invertida con su base hacia el cráneo y su vértice en el cuerno mayor del hueso hioides. Está separado rostralmente de la fosa infratemporal por el músculo pterigoideo medial y su fascia, caudalmente de la columna vertebral cervical por la

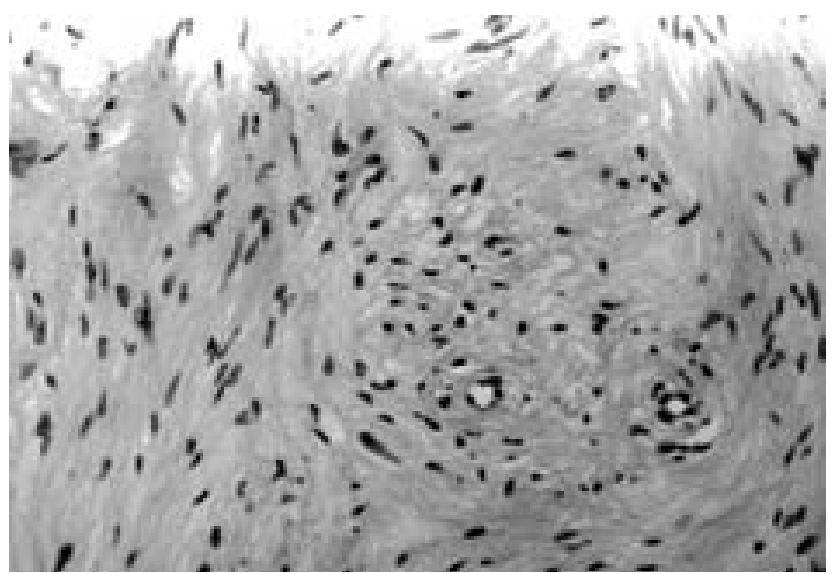

Figura 3 b. Detalle histopatológico.

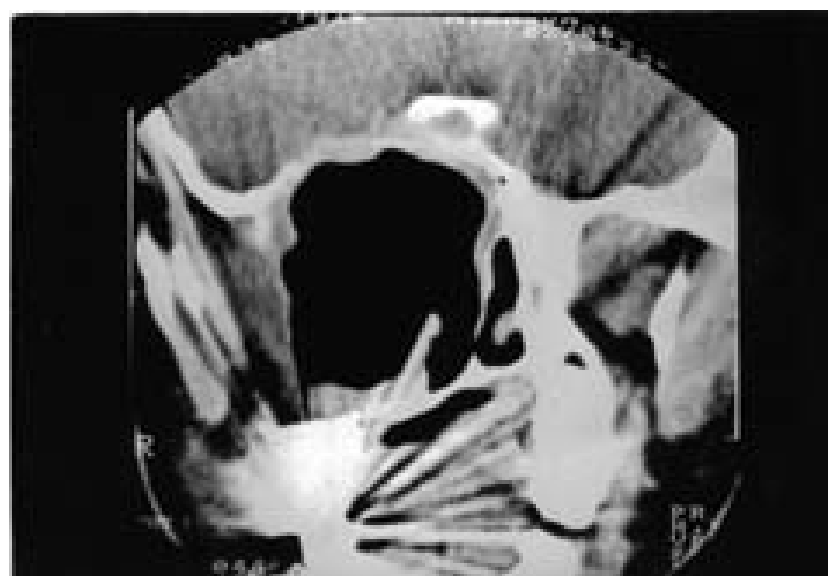

Figura 4 b. TC coronal que demuestra escaso residual paraselar.

fascia prevertebral y los músculos paravertebrales; medialmente de la nasofaringe por los músculos tensor del velo del paladar, constrictor superior de la faringe y la fascia faringobasilar. El EPF se divide en un compartimento preestiloideo y otro retroestiloideo por el diafragma estiloideo, constituido por los músculos estilofaríngeo, estilogloso y estilohioideo, y los ligamentos estilomaxilar y estilofaríngeo $^{6,13,14}$.

La fosa pterigopalatina (FPP) se encuentra limitada caudalmente por la apófisis pterigoides, medialmente por la lámina perpendicular del palatino, rostralmente por la pared posterior del seno maxilar; dorsalmente se abre al apex orbitario a través de la fisura orbitaria inferior y a la cavidad intracraneana a través del agujero redondo mayor, justo a 4 ó $5 \mathrm{~mm}$ ventrolateral de la fisura orbitaria ${ }^{6,9,13,14,31}$. Lateralmente, la FPP se comunica con la FIT mediante la hendidura pterigomaxilar, permitiendo el paso de la arteria maxilar interna.

Por sus características de benignidad lento crecimiento, los shwannomas intracraneales que se extienden 
Tabla 1

Casos de schwannomas trigeminales con extensión hacia la fosa infratemporal reportados en la literatura mundial.

\begin{tabular}{|c|l|c|c|c|}
\hline Pact. & \multicolumn{1}{|c|}{ Autor y año } & Referencia & Edad & Sexo \\
\hline 1 & Fleury y Furtado, 1955. & 12 & 51 & $\mathrm{~F}$ \\
\hline 2 & Lin y cols., 1974. & 17 & 54 & $\mathrm{M}$ \\
\hline 3 & Arena y Hilal, 1976. & 4 & 16 & $\mathrm{~F}$ \\
\hline 4 & Bitoh y cols., 1983. & 20 & 38 & $\mathrm{~F}$ \\
\hline 5 & Nager, 1984. & 7 & 25 & $\mathrm{M}$ \\
\hline 6 & Iwai y cols., 1988. & 16 & 7 & $\mathrm{~F}$ \\
\hline 7 & McCormick y cols.,1988 & 19 & 49 & $\mathrm{M}$ \\
\hline 8 & Lunardi y cols., 1989. & 18 & 55 & $\mathrm{~F}$ \\
\hline 9 & Pollack y cols., 1989. & 24 & 41 & $\mathrm{~F}$ \\
\hline 10 & Pollack y cols., 1989. & 24 & 58 & $\mathrm{~F}$ \\
\hline 11 & Beauvillain y cols. 1991 & 5 & 28 & $\mathrm{~F}$ \\
\hline 12 & Visot y cols., 1992. & 29 & 42 & $\mathrm{~F}$ \\
\hline 13 & Visot y cols., 1992. & 29 & 42 & $\mathrm{~F}$ \\
\hline 14 & Visot y cols., 1992. & 29 & 23 & $\mathrm{~F}$ \\
\hline 15 & Visot y cols., 1992. & 29 & 31 & $\mathrm{~F}$ \\
\hline 16 & Visot y cols., 1992. & 29 & 34 & $\mathrm{~F}$ \\
\hline 17 & Samii y cols., 1995. & 25 & 50 & $\mathrm{~F}$ \\
\hline 18 & Paquis y cols., 1998. & 23 & 36 & $\mathrm{M}$ \\
\hline 19 & Paquis y cols., 1998. & 23 & 60 & $\mathrm{M}$ \\
\hline 20 & Paquis y cols., 1998. & 23 & 63 & $\mathrm{~F}$ \\
\hline 21 & Yoshida y Kawase,1999 & 31 & 9 & $\mathrm{~F}$ \\
\hline 22 & Yoshida y Kawase,1999 & 31 & 58 & $\mathrm{~F}$ \\
\hline 23 & Yoshida y Kawase,1999 & 31 & 61 & $\mathrm{~F}$ \\
\hline 24 & Yoshida y Kawase,1999 & 31 & 25 & $\mathrm{~F}$ \\
\hline 25 & Yoshida y Kawase,1999 & 31 & 36 & $\mathrm{~F}$ \\
\hline 26 & Akhaddar y cols., 2002. & 3 & 35 & $\mathrm{~F}$ \\
\hline 27 & Presente caso & & 39 & $\mathrm{~F}$ \\
\hline
\end{tabular}

extracranealmente, pueden llegar a superar los $3 \mathrm{cms}$. de diámetro en el momento del diagnóstico ${ }^{3,31}$.

Por los casos ya descritos y por el nuestro, podemos evidenciar características importantes de los mismos. La media de edad en momento del diagnóstico es de 39.5 años, con un rango amplio que va desde los 7 a los 63 años, existiendo un franco predominio en el sexo femenino (22 pacientes/81.5\%). En lo que respecta a la sintomatología (Tabla 2), la duración desde el inicio hasta el diagnóstico se situa entre 9 días hasta 13 años, con una media de 24.6 meses y una moda de 18 meses, recalcando que nuestro caso fue el de mayor duración. Los síntomas y signos de afección al nervio trigémino, evidentemente, fueron los preponderantes en el 74\% (20 casos) y, de estos, el dolor facial ocurrió en $9(45 \%)$, hipoestesia en $6(30 \%)$, parestesias en $4(20 \%)$, y paresia de masticadores en 1 paciente $(5 \%)$. Nuestro caso no presentó ningún dato anormal referente a afección trigeminal, similar a otros 6 pacientes de la serie $(25.9 \%)$, pero debutó con cefalea, proptosis, diplopía y obstrucción nasal, que en la serie analizada correspondieron a $7.4 \%$, $14.8 \%, 18.5 \%$ y $11.1 \%$ respectivamente. El diagnóstico clínico se torna difícil; por lo tanto, las imágenes nos ayudan ampliamente. En la TC el tumor se presenta isodenso o ligeramente hiperdenso y suele realzarse intensamente con la administración de contraste; la ventana ósea nos muestra alteraciones de los huesos circundantes que, más 
Tabla 2

Clínica hallada en la serie de schwannomas con extensión hacia la fosa infratemporal.

\begin{tabular}{|l|c|}
\hline \multicolumn{1}{|c|}{ SINTOMAS Y SIGNOS } & No.(\%) \\
\hline a) Trigeminales: & $20(74 \%)$ \\
\hline Dolor & $9(45 \%)$ \\
\hline Hipoestesia & $6(30 \%)$ \\
\hline Parestesias & $4(20 \%)$ \\
\hline Paresia masticatoria & $1(5 \%)$ \\
\hline b) Diplopia: & $5(18.5 \%)$ \\
\hline c) Excitalmos: & $4(14.8 \%)$ \\
\hline d) Masa en mejilla: & $2(7.4 \%)$ \\
\hline e) Disminución de AV: & $3(11.1 \%)$ \\
\hline f) Obstrucción nasal: & $3(11.1 \%)$ \\
\hline g) Hipoacusia: & $3(11.1 \%)$ \\
\hline h) Cefalea: & $2(7.4 \%)$ \\
\hline i) Dolor otico: & $1(3.7 \%)$ \\
\hline d) Otitis media serosa: & $1(3.7 \%)$ \\
\hline
\end{tabular}

que erosionados y destruidos, se presentan desplazados por el tipo de crecimiento tumoral. En la Resonancia Magnética se muestran bien definidos y circunscritos, isointensos o hipointensos, en relación al parénquima cerebral, en T1 e hiperintenso en T2; sin embargo, la visualización de un componente intracraneal de la fosa media, extendiéndose hacia la fosa infratemporal, nos da la verdadera sospecha diagnóstica, que en el caso aquí descrito se complicó por no presentarse una evidencia clara del origen intracraneano en la RM. En la angiografía se muestran avasculares, con desplazamiento de vasos vecinos ${ }^{3}$.

Se han descrito algunas técnicas para el abordaje de la fosa infratemporal. Fisch describió tres tipos, donde básicamente el tipo $\mathrm{C}$ es el indicado para el tratamiento de lesiones tumorales que se extienden desde la región paraselar. El abordaje se realiza con una incisión similar a la indicada para una parotidectomía extendida, que se inicia a nivel de la carilla lateral del frontal y se extiende en sentido ventral, pasando sobre el arco cigomático y rostral al trago, diriéndose caudal al gonión y terminando en el cuello. La técnica consiste principalmente en una transposición permanente del nervio facial, resección del cóndilo mandibular, rechazo temporal del zigoma, resección de la apófisis pterigoides, destrucción de la trompa de Eustaquio, con una petrosectomía subtotal y obliteración del conducto auditivo medio, con exposición y eventual destrucción de la rama mandibular del trigémino. Con esta técnica se logra una exposición amplia, pero con desventajas importantes tales como disfunción facial, pérdida permanente de la audición conductiva, trastornos de la oclusión mandibular, alteraciones sensitivas faciales, además de potencial defecto cosmético ${ }^{11}$. El abordaje subtemporal-preauricular zigomático descrito por Sekhar y cols. difiere de la técnica de Fisch en que la incisión preauricular evita la pérdida de la audición y agrega la craneotomía frontotemporal

Tabla 3

Abordajes quirúrgicos empleados en los casos reportados en la literatura.

\begin{tabular}{|l|l|c|}
\hline \multicolumn{1}{|c|}{ ABORDAJES } & \multicolumn{1}{|c|}{ No.(\%) } & $\begin{array}{c}\text { Paciente } \\
\text { No. }\end{array}$ \\
\hline Subtemporal ID & $5(18.5 \%)$ & $7,8,12,13$ y 22 \\
\hline Zigomático infratemporal & $3(11.1 \%)$ & 23,24 y 25. \\
\hline Subtemporal ED transmaxilar & $3(11.1 \%)$ & 18,19 y 20. \\
\hline Subtemporal ID transmaxilar & $2(7.4 \%)$ & 4 y 11 \\
\hline Frontotemporal infratemporal & $2(7.4 \%)$ & 9 y 10 \\
\hline Ifratemporal ED & $2(7.4 \%)$ & 14 y 15. \\
\hline Subtemporal ED & $1(3.7 \%)$ & 5. \\
\hline Temporomandibular & $1(3.7 \%)$ & 1. \\
\hline Subtemporal ED transmandibular & $1(3.7 \%)$ & 2. \\
\hline Subtemporal Transzigomatico & $1(3.7 \%)$ & 16. \\
\hline Subtemporal ED e ID & $1(3.7 \%)$ & 26. \\
\hline Transzigomático & $1(3.7 \%)$ & 3. \\
\hline Orbitozigomático infratemporal & $1(3.7 \%)$ & 6. \\
\hline Frontotemporal ED & $1(3.7 \%)$ & 17. \\
\hline $\begin{array}{l}\text { Frontotemporal y destechamiento } \\
\text { de orbita }\end{array}$ & $1(3.7 \%)$ & 21. \\
\hline $\begin{array}{l}\text { Centrofacial } \\
\text { transmaxilar }\end{array}$ & $1(3.7 \%)$ & 27. \\
\hline
\end{tabular}


mejorando la exposición en lesiones amplias y con componente intracraneano importante; cuenta con desventajas primordiales, como la potencial fístula de LCR requiriendo de drenaje de LCR durante 3 a 5 días, el potencial defecto cosmético, alteraciones sensitivas faciales y la necesidad, en ocasiones de traqueostomía ${ }^{27}$. Al Mefty y Anand describieron una modificación del abordaje frontotemporal preauricular con osteotomía de apófisis coronoides, destacándose la excelente exposición de la FIT que se consigue con el levantamiento del tendón del músculo temporal; sin embargo no estuvieron libres de trastornos en la apertura mandibular y de problemas cosméticos ${ }^{1}$. Guinto y cols. agregaron la osteotomía de la rama ascendente del hueso mandibular con gran exposición, pero sin encontrarse exentos de complicaciones similares a las mencionadas en técnicas precursoras ${ }^{13}$. Catalano y Biller proponen la maxilotomía extendida mediante incisión cutánea de Weber-Ferguson extendida y asociada a una incisión frontotemporal, con gran exposición y buen manejo de las estructuras vasculares de la base del cráneo; sin embargo, puede requerir la traqueostomía y sus complicaciones potenciales son el ectropión, alteraciones cosméticas y contaminación intracraneal, por exposición de las mucosas ${ }^{8,22}$. Hitsomatsu y Rothon proponen la maxilectomía superior e inferior que ellos realizaron en cadáveres ${ }^{14}$.

Muchos de los abordajes mencionados suelen requerir la colaboración de un equipo de cirujanos maxilofaciales, para optimizar resultados y disminuir el tiempo de exposición quirúrgica. Todos los pacientes de la serie analizada fueron sometidos a cirugía (Tabla 3). En el caso que presentamos, el escaso componente intracraneano, la extensión de la lesión hacia órbita, espacio parafaríngeo, seno maxilar superior y fosas nasales, además de la fosa infratemporal, hizo que no recurriéramos a craneotomías convencionales $\mathrm{y}$, por la ausencia de un servicio de cirugía maxilofacial en nuestra institución, nos abstuvimos de maxilectomía y abordajes transmandibulares. Practicamos un abordaje intercartilaginoso centrofacial transmaxilar, el que no fue utilizado en los demás casos. Dentro de las complicaciones postoperatorias, dos $(7.4 \%)$ pacientes presentaron neuroinfección y 2 más $(7.4 \%)$ cursaron con fístula de líquido cefalo-raquídeo y un paciente falleció por contusión temporal. En nuestro caso no se presentaron complicaciones, sin embargo la exéresis fue subtotal. Se ha reportado que la exéresis completa de los schwannomas con estas características solamente es posible en el $50 \%$ de los $\operatorname{casos}^{3,23,25}$.

\section{Conclusiones}

Los schwannomas trigeminales con extensión hacia la fosa infratemporal son entidades infrecuentes. El caso presentado por nosotros tiene características peculiares, en comparación a los descritos previamente. Los más destaca- dos son: su escaso componente intracraneal, y la afectación de la órbita, espacio parafaríngeo, seno maxilar y fosas nasales. Las condiciones mencionadas son precisamente las que orientaron nuestra decisión quirúrgica, por lo tanto consideramos que el abordaje intercartilaginoso-centrofacial-transmaxilar, con exéresis intracapsular, puede ser adecuado para este tipo de tumores con ausencia de fístula de LCR y contaminación del contenido intracraneal por la no apertura dural; sin embargo, no se encuentra libre de desventajas, como es el escaso control y visualización de las estructuras. Es menester recalcar que, a pesar de que prescindimos de ella en el presente caso, la angiografía preoperatoria debe ser una herramienta importante en la planificación quirúrgica, para evitar complicaciones vasculares, (si tomamos en cuenta que en la fosa infratemporal transcurren la arteria maxilar interna y sus ramas), a pesar de ser un tumor escasamente vascularizado.

\section{Bibliografía}

1. Al-Mefty, O., Anand, V.K.: Zygomatic approach to skull base lesions. J Neurosurg 1990; 73: 668-673.

2. Al-Mefty, O., Ayoubi, S., Gaber, E.: Trigeminal schwannomas. Removal of dumbell shaped tumors through the expanden Meckel's cave and outcomes of cranial nerve function. J Neurosurg 2002; 96: 453-463.

3. Akhaddar, A., El Mostarchid, B., Zrara, I., Boucetta, M.: Intracranial Trigeminal neuroma involving the infratemporal fossa: Case report an review of the literature. Neurosurgery 2002; 50: 633-638.

4. Arena, S., Hilal, E.Y.: Neurilemomas of the in fratemporal space : Report of a case and review of the literature. Arch Otolaryngol 1976; 102: 180184

5. Beauvillain, C., Calais, C., Launay, M.C., Bordure, P., Zanaret, M., Legent, F.: Nervous tumors of the infratemporal fossa. Ann Otolaryngol Chir Cervicofac 1991; 108: 107-111.

6. Bejjani, G.K., Sullivan, B., Salas-López, E., Abello, J., Wright, D.C., Jurjus, A., Sekhar, L.N.: Surgical anatomy of the infratemporal fossa: The styloid diaphragm revisited. Neurosurgery 1998; 43: 842-853.

7. Bitoh, S., Hasegawa, H., Ohtsuki, H., Obashi, J., Furukawa, Y., Sakurai, M.: Schwannoma of the skull base with intracranial extension. Surg Neurol 1983; 20: 143-146.

8. Catalano, P.J., Biller, H.F.: Extended osteoplastic maxillotomy. A versatile new procedure for wide access to central skull base and infratemporal fossa. Arch Otyolaryngol Head Neck Surg 1993; 119: 394-400.

9. Couldwell, W.T., Sabit, I.B., Weiss, M.H., Giannotta, S.L., Rice, D.: Transmaxillary approach to anterior cavernous sinus: A Microanatomic study surgical approaches. Neurosurgery 1997 ; 40: 1307-1311.

10. Ezura, M., Ikedi, H., Ogawa, A., Yoshimoto, T.: Intracerebral Schwannoma: Case report. Neurosurgery 1992; 
30: 97-100.

11. Fisch,U.: Infratemporal fossa approach of tumors of the temporal bone and base skull. J Laringol Otol 1978; 92: 949-967.

12. Fleury, P., Furtado, R.: Deux cas de neurinomes e la fosse ptérigomaxillaire. Ann Otolaryngol Chir Cervicofac 1955; 72 : 920-925.

13. Guinto, G., Abello, J., Molina, A., Gallegos, F., Oviedo, A., Nettel, B., López, R.: Zygomatic-transmandibular approach for giant tumors of the infratemporal fossa an parapharyngeal space. Neurosurgery 1999; 45: 1385-1398.

14. Hitotsumatsu, T., Rhoton, AL.: Unilateral upper an lower subtotal maxillectomy approaches to cranial base: Microsurgical Anatomy. Neurosurgery 2000; 46: 14161453.

15. Huang, P., Zagzag, D., Benjamin, V.: Intracranial schwannoma presenting as a subfrontal tumor: Case report. Neurosurgery 1997; 40: 194-197.

16. Iwai, Y., Hakuba, A., Noguchi, K., Nishimura, S.: A gigantic neurilemoma originating in the pteryopalatine fossa: case report. Surg Neurol 1988; 30: 452-456.

17. Lin, S.R., Lin, S.Z., Tatoian, J.A.: Trigeminal neurinoma with extracvaranial extension. Neuroradiology 1974; $8: 183-185$.

18. Lunardi, P., Missori, P., Gagliardi, F.M., Fraioli, B.: Trigeminal schwannoma with infratemporal extension: Case report. J Neurosurg Sci 1989; 33: 293-295.

19. McCormick, P.C., Bello, J.A., Post, K.D.: Trigeminal schwannoma: Surgical series of 14 cases with review of the literature. J Neurosurg 1988; 69: 850-860.

20. Nager, E.T.: Neurinomas of the trigeminal nerve. Am J Otolaryngol 1984; 5: 301-333.

21.Oikawa, A., Takeda, N., Aoki, N., Takizawa, T., Sakoma, T.: Scwannoma arising from the tentorium at an unusual location: Case report. Neurosurgery 2002; 50: 1352-1355.

22. Osborne, E., Clayton, M., Fenwick, J.D.: The leeds modified Webwr-Ferguson incision. Laryngol Otol 1987; 101: 465-466.
23. Paquis, P., Castillo, L., Lonjon, M., Santini, J., Grellier, P.: Extracranial trigeminal schwannomas with middle temporal fossa development. Neurochirurgie 1998; 44: 38-45.

24. Pollack, I.F., Sekhar, L.N., Jannetta, P.J., Janecka, I.P.: Neurilemomas of the trigeminal nerve. J Neurosurg 1989; 70: 737-745.

25. Samii, M., Migliori, M.M., Tatagiba, M., Babu, R.: Surgical treatment of trigeminal schwannomas. J Neurosurg 1995; 82: 711-718.

26. Sarma, S., Sekhar, L.N., Schessel, D.A.: Nonvestibular schwannomas of the brain: A 7-year experience. Neurosurgery 2002; 50: 437-449.

27. Sekhar, L.N., Schramm, V.L., Jones, F.J.: Subtemporalpreuricular infratemporal fossa approach to large lateral and posterior cranial base neoplasms. J Neurosurg 1987; 67: 488499.

28.Tedeshi, H., Rhoton, J.A. Jr.: Lateral approaches to the petroclival region. Surg Neurol 1994; 41: 180-216.

29. Visot, A., Derome, P., De Leon, J.: Sphenocavernous and infratemporal trigeminal neurinomas : Surgical series of 15 cases. Skull Base Surg 1992; 3: 142-149.

30. Vrionis, F.D., Cano, W.G., Heilman, C.B.: Microsurgical anatomy of the infratemporal fossa as viewed laterally and superiorly surgical anatomy. Neurosurgery 1996; 39: 777786.

31. Yoshida, K., Kawase, T.: Trigeminal neurinomas extending into multiple fossae: Surgical methods and review of the literature. J Neurosurg 1999; 91: 202-211.

Santos-Franco, J.A.; Barragán, A.; Mercado-Pimentel, R; Ortiz-Velásquez, I.; García-Pastor, C.; Barquet-Platón, E.I.; Pane-Pianesse C.; Gómez-Llata, S.: Schwannoma trigeminal intracraneal con extensión a la fosa infratemporal, espacio parafaríngeo, órbita, seno maxilar y fosa nasal. A propósito de un caso. Neurocirugía 2005; 16: 67-74.

Correspondencia postal: J.A. Santos Franco. Servicio de Neurocirugía. Instituto Nacional de Neurología y Neurocirugía "Manuel Velasco Suárez". México. 